TITLE:

\title{
Dielectric properties of microvillous cells simulated by the three- dimensional finite-element method
}

$\operatorname{AUTHOR}(S):$

Asami, Koji

\section{CITATION:}

Asami, Koji. Dielectric properties of microvillous cells simulated by the three-dimensional finite-element method. Bioelectrochemistry 2011, 81(1): 28-33

ISSUE DATE:

2011-04

URL:

http://hdl.handle.net/2433/139498

\section{RIGHT:}

(c) 2011 Elsevier B.V.; この論文は出版社版でありません。引用の際には 出版社版をご確認ご利用ください。; This is not the published version. Please cite only the published version. 


\title{
Dielectric properties of microvillous cells simulated by three-dimensional finite-element method
}

Koji Asami

Institute for Chemical Research, Kyoto University, Uji, Kyoto 611-0011, Japan

Corresponding author: Koji Asami

Institute for Chemical Research, Kyoto University, Uji, Kyoto 611-0011, Japan

Tel: 81-774-38-3081, Fax: 81-774-38-3084

E-mail: asami@e.kuicr.kyoto-u.ac.jp

\begin{abstract}
Most of biological cells have microvilli on their surfaces, which significantly influence their dielectric properties. The complex permittivity of a cubical system containing a spherical cell model with cylindrical projections was calculated over a frequency range of $10 \mathrm{kHz}$ to $100 \mathrm{MHz}$ using three-dimensional finite-element method. The spectra of the complex permittivity consisted of low- and high-frequency relaxation processes which were respectively attributed to the polarization of the membranes covering the projections and the spherical body. Conventional analysis based on the spherical shell model was applied to the simulated spectra to discuss effects of cell surface morphology on the electric parameters estimated for the plasma membrane and the cytoplasm.
\end{abstract}

Keywords: Interfacial polarization; Dielectric spectroscopy; Biological cell; Microvillus; Simulation.

\section{Introduction}

Biological cells are polarized in electric fields owing to interfacial polarization, which behavior has been studied by dielectric spectroscopy (DS) and the electrokinetic methods of dielectrophoresis (DEP) and electrorotation (ER). DS measures the complex relative permittivity $\varepsilon^{*}$ of a cell suspension as a function of the frequency $f$ of the applied ac field. Complex relative permittivity is defined as $\varepsilon^{*}=\varepsilon$-j $\kappa \omega \varepsilon_{0}$, where $\varepsilon$ is relative permittivity, $\kappa$ electric conductivity, $\omega=2 \pi f, \varepsilon_{0}$ the permittivity of vacuum and $j^{2}=-1$. On the other hand, DEP and ER respectively measure the force and torque that a single cell experiences in heterogeneous ac fields and rotating fields. The force and torque are related to the polarizability or the Clausius-Mossotti factor $K$ of the cell. The spectra of $\varepsilon^{*}$ and $K$ have been usually analyzed by theories based on the spherical shell model that is a sphere covered with a thin shell to estimate the electric parameters of cell components such as membrane capacitance $C_{\mathrm{m}}$ and cytoplasmic conductivity $\kappa_{\mathrm{i}}$ [1-7]. For a spherical cell with a smooth surface, $C_{\mathrm{m}}$ is the specific membrane capacitance defined by $C_{\mathrm{m}}=\varepsilon_{\mathrm{m}} \varepsilon_{0} / d$ and the values of 6-8 $\mathrm{mF}$ $\mathrm{m}^{-2}$ have been obtained for cells with smooth surfaces like erythrocytes [8-13]. However, larger $C_{\mathrm{m}}$-values have been obtained for many cultured cells, being mainly due to the presence of 
membrane-bound projections that are extensions of the plasma membrane [14-22]. Irimajiri et al. [14] studied dielectric properties of basophil leukaemia cells whose volume was changed by osmotic perturbation, and clearly demonstrated that the estimated value of $C_{\mathrm{m}}$ changing with the cell radius $R$ correlated to the "microvilation" or the surface roughness of the cells. Similar studies were made with mouse myeloma and hybridoma cells [15] and murine erythroleukaemia cells [16] by ER, suggesting that the cell total membrane capacitance $C_{\mathrm{t}}$ defined by $C_{\mathrm{t}}=4 \pi R^{2} C_{\mathrm{m}}$ was kept constant during osmotic perturbation, i.e., $C_{\mathrm{t}}$ was independent of $R$. Further, the correlation between the value of $C_{\mathrm{m}}$ and the "microvilation" was found for cells responding to apoptosis-inducers and toxicants [17-22]. In contrast to the experimental studies, however, there are few theoretical studies about the effects of the cell surface morphology on the dielectric properties of cells.

For simple cell models such as spherical and ellipsoidal shell models, we can use analytical equations that relate the electric and morphological parameters of the cell components to the dielectric properties of cells and cell suspensions. For cells possessing complex structures, however, analytical equations are not available, and therefore numerical simulation techniques are required. Recently, various numerical techniques have been developed to simulate dielectric properties of biological cells, enabling us to deal with more realistic cell models [23-37].

This paper is concerned with numerical simulation of the dielectric properties of a cell model that emulates the morphological changes of microvilli during osmotic perturbation. Microvilli are most common membrane-bound projections of a few hundreds nanometers in diameter [38]. The length and number of microvilli change in response to environmental stimuli and cellular conditions such as osmotic perturbation, apoptosis and differentiation. In the simulation, a microvillous cell is modeled as a sphere with cylindrical projections. Electric potential distributions in a cubic box containing the cell model in a medium are numerically solved by the three-dimensional finite-element method (FEM), providing the $\varepsilon^{*}$ of the system as a function of $f$. Since experimental $\varepsilon^{*}$ spectra have been commonly analyzed using the spherical shell model irrespective of the presence of microvilli, the same analysis is applied to the simulated spectra to discuss relationships between the estimated values of $C_{\mathrm{m}}$ and $\kappa_{\mathrm{i}}$ and the cell surface morphology.

\section{Methods}

\subsection{Cell Models}

A microvillous cell is modeled as a sphere of radius $R_{\mathrm{s}}$ with $n_{\mathrm{p}}$ cylindrical projections of radius $r_{\mathrm{p}}$ and length $h$ (Fig. 1). To simplify the morphological change of the cell during osmotic perturbation, it is assumed that the projections of a constant value of $n_{\mathrm{p}}$ increase in $h$ with decreasing the cell volume $V_{\mathrm{c}}$ while keeping the total cell surface area $S_{\mathrm{c}}$ constant. $S_{\mathrm{c}}$ and $V_{\mathrm{c}}$ are approximately given by Eqs. (1) and (2), respectively, and $S_{\mathrm{c}}$ is equal to that of a sphere of radius $R_{0}$ without projection.

$$
\begin{aligned}
S_{c} & =2 \pi\left(2 R_{s}^{2}+n_{p} r_{p} h\right)=4 \pi R_{0}^{2}, \\
V_{c} & =\frac{4}{3} \pi R_{s}^{3}+n_{p} \pi r_{p}^{2} h .
\end{aligned}
$$


The surface area ratio $S_{\mathrm{p}}$ of the projections to the whole cell becomes $S_{p}=\pi n_{p}\left(r_{p}^{2}+2 r_{p} h\right) /\left(4 \pi R_{0}^{2}\right)$.

The cell model is placed at the center of a cubic box of $L \times L \times L$, whose top (at $z=+L / 2$ ) and bottom (at $z=-L / 2$ ) correspond to electrodes. Since the cell model is symmetrical about each of the vertical planes of $x=0$ and $y=0$, the domain subjected to numerical calculation can be confined to a quarter of the cubic box (Fig. 1A). The quarter is also symmetrical about the horizontal plane of $z=0$, and therefore, when voltages of $+1 \mathrm{~V}$ and $-1 \mathrm{~V}$ are respectively applied to the top and bottom electrodes, the middle horizontal plane (at $z=0$ ) becomes an equipotential surface of $0 \mathrm{~V}$. This further allows us to confine the numerical calculation to a half of the quarter.
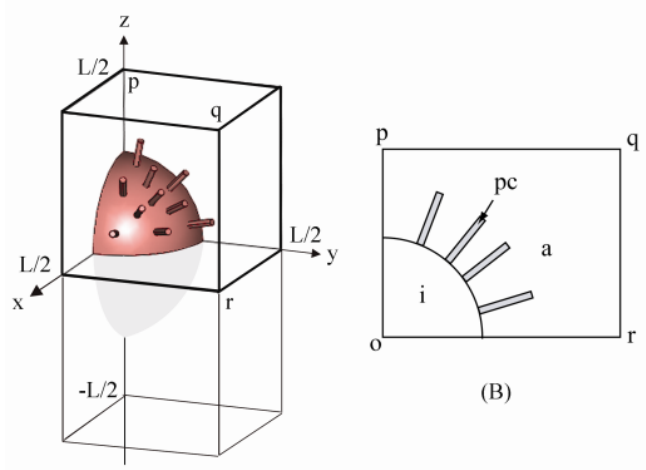

(B)

(A)

Fig. 1 (A) A cell model with cylindrical projections in a cubic box. The eighth part of the cubic box, which is rimmed with thick lines, is used for actual numerical simulation because of symmetrical geometry. The illustration corresponds to model $b$ with $R_{\mathrm{s}}=4 \mu \mathrm{m}, r_{\mathrm{p}}=0.15 \mu \mathrm{m}$ and $n_{\mathrm{p}}=80$ in a cube of $L=16 \mu \mathrm{m}$. (B) A cross-section through points $p, q$ and $r$ in the cell model is used for cross-sectional views of the electric potential distributions shown in Fig. 6. Point $o$ is the origin of the coordinate, and subdomains $p c, i$ and $a$ indicate the projection core, cytoplasm and external medium, respectively.

\subsection{Numerical calculation}

Numerical calculation was made by FEM using Comsol Multiphysics with an AC/DC Module (COMSOL $\mathrm{AB})$ in quasi electrostatic and time harmonics modes over a frequency range from $1 \mathrm{kHz}$ to $1 \mathrm{GHz}$. Biological cells possess thin plasma membranes, which require extra fine meshes and thus considerable computation tasks. To solve the problem, the "thin-layer" approximation that deals with the membrane as an interface of zero thickness was adopted in a previous paper [37]. The same technique was used for this calculation. We consider subdomains $i, a$ and $p c$ that respectively correspond to the cell interior, the external medium and the projection core (see Fig.1)). The subdomains $i, a$ and $p c$ have complex relative permittivities $\varepsilon_{\mathrm{i}}{ }^{*}, \varepsilon_{\mathrm{a}}{ }^{*}$ and $\varepsilon_{\mathrm{pc}}{ }^{*}$, respectively. Both interfaces between the subdomains $i$ and $a$ (interface $i-a$ ) and between the subdomains $p c$ and $a$ (interface $p c-a$ ) have the specific complex capacitance of $\varepsilon_{0} \varepsilon_{\mathrm{m}}{ }^{*} / d$, where $d$ is the membrane thickness and $\varepsilon_{\mathrm{m}}{ }^{*}$ is the complex relative permittivity of the membrane. As described in section 2.1, numerical calculation was performed for the eighth part of the model system, where distributions of 
electric potential $\phi$ were solved under the following boundary conditions. (i) For the four sides of the cubic domain, electric flux density is zero. (ii) The top and the bottom of the cubic domain have potentials of 1 and $0 \mathrm{~V}$, respectively. (iii) The interfaces $i$ - $a$ and $p c-a$ have potential differences and respectively have electric flux densities $D$

$$
D=-n_{i} \cdot \varepsilon_{i}^{*} \varepsilon_{0} \nabla \phi_{i}=n_{a} \cdot \varepsilon_{a}^{*} \varepsilon_{0} \nabla \phi_{a}=\frac{\varepsilon_{m}^{*} \varepsilon_{0}}{d}\left(\phi_{a}-\phi_{i}\right)
$$

and

$$
D=-n_{p c} \cdot \varepsilon_{p c}^{*} \varepsilon_{0} \nabla \phi_{i}=n_{a} \cdot \varepsilon_{a}^{*} \varepsilon_{0} \nabla \phi_{a}=\frac{\varepsilon_{m}^{*} \varepsilon_{0}}{d}\left(\phi_{a}-\phi_{p c}\right)
$$

where $n_{\mathrm{i}}, n_{\mathrm{a}}$ and $n_{\mathrm{pc}}$ are outward normal unit vectors to the boundaries of subdomains $i, a$ and $p c$, and $\phi_{1}, \phi_{\mathrm{a}}$ and $\phi_{\mathrm{a}}$ are the electric potentials of subdomains $i, a$ and $p c$ at the interfaces. (iv) The interface $p c-i$ has no potential difference and holds for continuity of electric flux density as

$$
-n_{p c} \cdot \varepsilon_{p c}^{*} \varepsilon_{0} \nabla \phi_{p c}=n_{i} \cdot \varepsilon_{i}^{*} \varepsilon_{0} \nabla \phi_{i}
$$

The free charge on the top boundary of the cube is calculated by integrating electric flux density over the boundary. The free charge and the potential difference between the top and the bottom boundaries provide the complex capacitance $C^{*}$ of the cube of $L / 2 \times L / 2 \times L / 2$, which is simply converted to the effective complex relative permittivity $\varepsilon^{*}$ as $\varepsilon^{*}=C^{*} /\left(\varepsilon_{0} L / 2\right)$.

\subsection{Determination of dielectric relaxation parameters}

The frequency dependence of the $\varepsilon^{*}$ obtained by numerical simulation was represented by

$$
\varepsilon^{*}=\varepsilon_{h}+\frac{\Delta \varepsilon_{h}}{1+\left(j f / f_{c h}\right)^{\beta_{h}}}+\frac{\Delta \varepsilon_{l}}{1+\left(j f / f_{c l}\right)^{\beta_{l}}}+\frac{\kappa_{l}}{j 2 \pi f \varepsilon_{0}},
$$

where $\varepsilon_{\mathrm{h}}$ is the high-frequency limit of relative permittivity, $\Delta \varepsilon$ the relaxation intensity, $f_{\mathrm{c}}$ the characteristic frequency, $\beta$ the Cole-Cole parameter $(0<\beta \leq 1), \kappa_{1}$ the low-frequency limit of conductivity, and subscripts $\mathrm{h}$ and 1 of $\Delta \varepsilon, f_{\mathrm{c}}$ and $\beta$ are referred to the high- and low-frequency relaxation processes. The relaxation parameters $\varepsilon_{\mathrm{h}}, \Delta \varepsilon_{\mathrm{h}}, f_{\mathrm{ch}}, \beta_{\mathrm{h}}, \Delta \varepsilon_{\mathrm{l}}, f_{\mathrm{cl}}, \beta_{1}$ and $\kappa_{1}$ were determined by fitting Eq. (6) to the simulated $\varepsilon^{*}$ spectra.

\section{Results and discussion}

\subsection{Dielectric spectra of suspensions of cells with projections}

Numerical simulation has been carried out using a cell model that is a sphere with cylindrical projections. To emulate dielectric behavior of microvillous cells in osmotic perturbation, the following conditions and geometric parameter values were adopted. (i) The cell surface area $S_{\mathrm{c}}$ is constant irrespective of changes in cell volume $V_{\mathrm{c}}$ and is equal to that of a sphere of $R_{0}=5 \mu \mathrm{m}$ in radius $\left(S_{\mathrm{c}}=314.2 \mu \mathrm{m}^{2}\right)$. (ii) The number $n_{\mathrm{p}}$ and radius $r_{\mathrm{p}}$ of the projections are respectively fixed at 
80 and $0.15 \mu \mathrm{m}$. (iii) The radius $R_{\mathrm{s}}$ of the spherical body and the length $h$ of the projections are both changed with $V_{\mathrm{c}}$. The geometric parameters of representative cell models are listed in Table 1 . The volume fraction $P_{\mathrm{g}}$ of the cell model occupied in a cubic box of $L=16 \mu \mathrm{m}$ is calculated by $P_{\mathrm{g}}=V_{\mathrm{c}} / L^{3}$. The equivalent cell radius $R_{\text {eq }}$ was calculated from the cell volume $V_{\mathrm{c}}$ assuming a corresponding sphere as $R_{\mathrm{eq}}=\left(3 V_{\mathrm{c}} / 4 \pi\right)^{1 / 3}$.

Table 1 Geometric parameters of representative cell models.

\begin{tabular}{|c|c|c|c|c|c|}
\hline Models & $R_{\mathrm{s}} / \mu \mathrm{m}$ & $h / \mu \mathrm{m}$ & $V_{\mathrm{c}} / \mu \mathrm{m}^{3}$ & $P_{\mathrm{g}}$ & $R_{\mathrm{eq}} / \mu \mathrm{m}$ \\
\hline $\mathrm{a}$ & 5.0 & 0 & 523.6 & 0.1278 & 5.00 \\
\hline b & 4.4 & 0.94 & 362.1 & 0.0884 & 4.42 \\
\hline c & 4.0 & 1.50 & 276.6 & 0.0675 & 4.04 \\
\hline d & 3.5 & 2.13 & 191.6 & 0.0468 & 3.58 \\
\hline
\end{tabular}

For sake of simplicity, numerical simulation was first carried out for the case of $\varepsilon_{\mathrm{i}}{ }^{*}=\varepsilon_{\mathrm{pc}}{ }^{*}$, using the following parameter values relevant to mammalian cells as: $\varepsilon_{\mathrm{a}}=\varepsilon_{\mathrm{i}}=\varepsilon_{\mathrm{pc}}=80, \quad \kappa_{\mathrm{a}}=1 \mathrm{~S} \mathrm{~m}^{-1}$, $\kappa_{\mathrm{i}}=\kappa_{\mathrm{pc}}=0.5 \mathrm{~S} \mathrm{~m}^{-1}, \varepsilon_{\mathrm{m}}=5, \kappa_{\mathrm{m}}=0 \mathrm{~S} \mathrm{~m}^{-1}$ and $d=5 \mathrm{~nm}$. Simulation for the case of $\varepsilon_{\mathrm{i}}^{*} \neq \varepsilon_{\mathrm{pc}}{ }^{*}$ will be discussed in section 3.2. Figure 2 shows frequency dependence of the relative permittivity $\varepsilon^{\prime}\left(\varepsilon^{\prime}=\varepsilon\right)$ and loss factor $\varepsilon^{\prime \prime}$ of a cubic system containing one of cell models $a$ - $d$. The value of $\varepsilon^{\prime \prime}$ was calculated by subtracting the low-frequency limit of conductivity $\kappa_{1}$ as $\varepsilon^{\prime \prime}=\left(\kappa-\kappa_{1}\right) /\left(2 \pi f \varepsilon_{0}\right)$. The dielectric spectra for a spherical model without projection (model $a$ ) showed dielectric relaxation with a single relaxation time, being in good agreement with those calculated from the Pauly-Schwan (PS) theory based on the spherical shell model [6]. On the other hand, cell models with projections (models $b$ - $d$ ) showed broader spectra containing two relaxation processes, which were well represented by Eq. (6) to provide the dielectric relaxation parameters of the low-frequency $(\mathrm{LF})$ relaxation $\left(\Delta \varepsilon_{\mathrm{l}}, f_{\mathrm{cl}}, \beta_{\mathrm{l}}\right)$ and the high-frequency $(\mathrm{HF})$ relaxation $\left(\Delta \varepsilon_{\mathrm{h}}, f_{\mathrm{ch}}, \beta_{\mathrm{h}}\right)$.

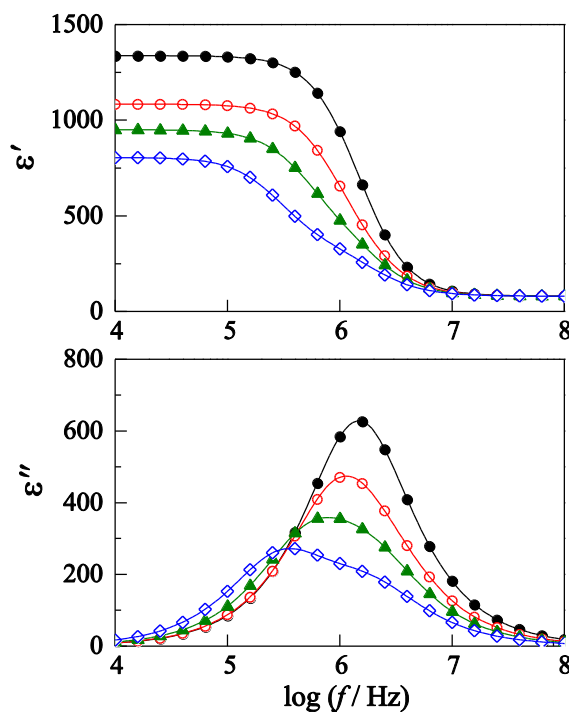


Fig. 2 The relative permittivity $\varepsilon^{\prime}$ and loss factor $\varepsilon^{\prime \prime}$ of a cubic system containing a cell model as a function of frequency $f$. Data points $(\bullet),(\circ),(\boldsymbol{\Delta})$ and $(\diamond)$ respectively correspond to models $a$ - $d$ given in Table 1 . Curves are the best-fit ones calculated from Eq. (6).

The dielectric relaxation parameters were plotted for the equivalent cell radius $R_{\text {eq }}$ in Figs. 3-5. It should be noted that data points at $R_{\text {eq }}=4.42$ and $4.71 \mu \mathrm{m}$ included large errors because the values of $f_{\mathrm{cl}}$ and $f_{\mathrm{ch}}$ were close to each other. In Fig. $3, \Delta \varepsilon\left(\Delta \varepsilon=\Delta \varepsilon \mathrm{l}+\Delta \varepsilon_{\mathrm{h}}\right)$ and $\Delta \varepsilon_{\mathrm{h}}$ decrease with decreasing $R_{\text {eq }}$ while $\Delta \varepsilon_{1}$ slightly changes. The ratio $\Delta \varepsilon_{1} / \Delta \varepsilon$ is, however, proportional to the surface area ratio $S_{\mathrm{p}}$ of the projections to the whole cell (see the inset of Fig. 3), suggesting the contribution of the projections to the LF relaxation. The $f_{\mathrm{cl}}$ and $f_{\mathrm{ch}}$ are separated by one decade at $R_{\text {eq }}$ of about $3 \mu \mathrm{m}$, and approach to each other with increasing $R_{\text {eq }}$ and merges into the characteristic frequency of the model without projection at $R_{\text {eq }}=5 \mu \mathrm{m}$ (Fig. 4). The Cole-Cole parameter $\beta$ determines the shape of dielectric relaxation. The decrease in $\beta_{\mathrm{h}}$ with decreasing $R_{\mathrm{eq}}$ indicates broadening of the $\mathrm{HF}$ relaxation, while the values of $\beta_{1}$ are almost unity, i.e., the Debye type relaxation for the LF relaxation (Fig. 5). At low $R_{\text {eq }}$, in addition to the broadening of the HF relaxation, a large difference between $f_{\mathrm{cl}}$ and $f_{\mathrm{ch}}$ results in broadening of the whole spectrum.

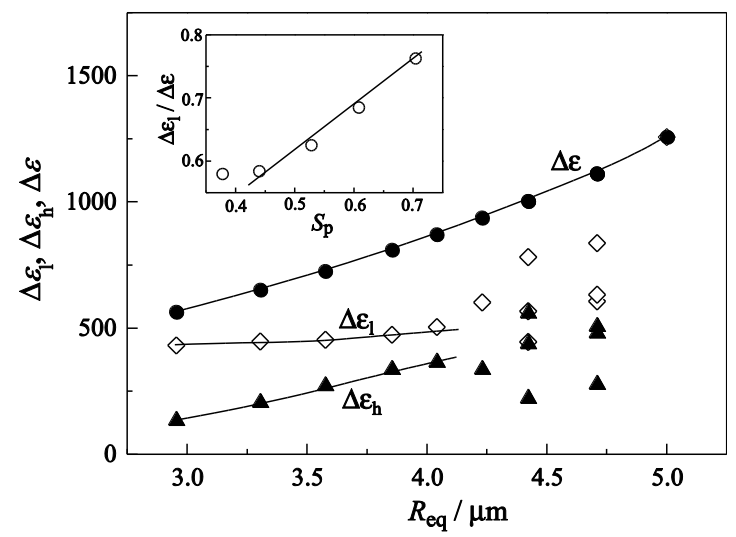

Fig. 3 Dielectric relaxation intensities $\Delta \varepsilon_{\mathrm{l}}(\diamond), \Delta \varepsilon_{\mathrm{h}}(\boldsymbol{\Delta})$, and $\Delta \varepsilon(\bullet)$ as a function of equivalent cell radius $R_{\mathrm{eq}}$. Inset: $\Delta \varepsilon_{\mathrm{l}} / \Delta \varepsilon$ plotted against the relative surface area $S_{\mathrm{p}}$ of the projections to the whole cell. Curves are a guide to eye.

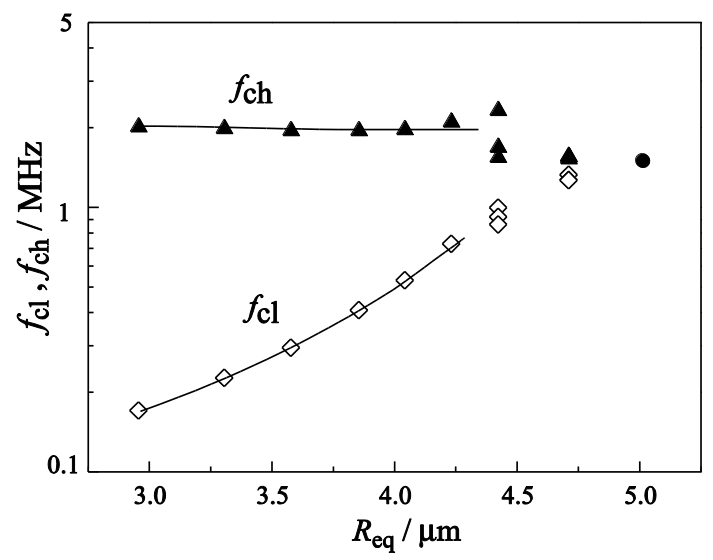

Fig. 4 Characteristic frequencies $f_{\mathrm{cl}}(\diamond)$ and $f_{\text {ch }}(\boldsymbol{\Delta})$ as a function of equivalent cell radius $R_{\text {eq }}$. The point $(\bullet)$ at $R_{\text {eq }}=5 \mu \mathrm{m}$ refers to the model without projection $(\operatorname{model} a)$. Curves are a guide to eye. 


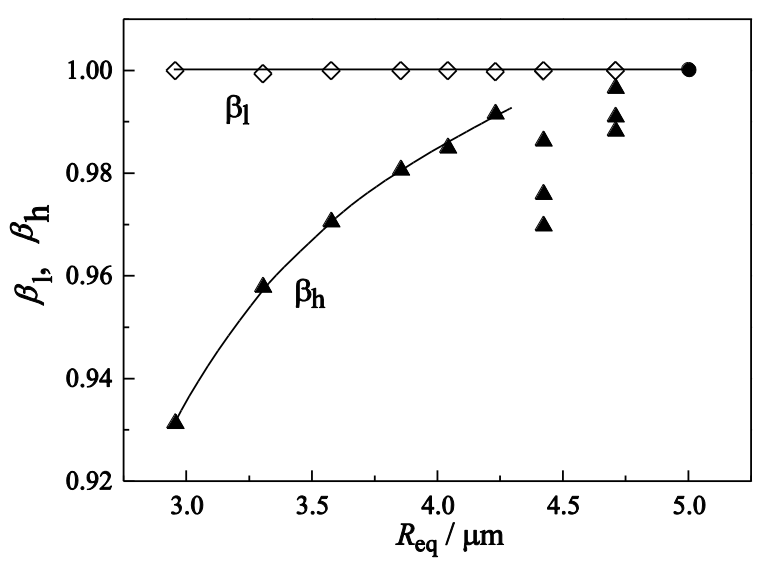

Fig. 5 Cole-Cole parameters $\beta_{1}(\diamond)$ and $\beta_{\mathrm{h}}(\boldsymbol{\Delta})$ as a function of equivalent cell radius $R_{\text {eq }}$. The point $(\bullet)$ at $R_{\text {eq }}=5 \mu \mathrm{m}$ refers to the model without projection. Curves are a guide to eye.

The LF and HF relaxation processes might be due to the membrane polarization of the projections and the spherical body, respectively. To assign the two relaxation processes, we examine electric potential distributions in the system at different frequencies. Figure 6 shows the distributions of the real part of electric potential for model $d$. The insides of the projections and the spherical body both have a constant potential below $100 \mathrm{kHz}$ and are clearly distinguished from the surroundings, indicating that the membranes of the projections and the spherical body are both fully polarized. At $1 \mathrm{MHz}$, where the LF relaxation diminishes, the projections become invisible and the projection membrane is less polarized. Finally the spherical body disappears at $10 \mathrm{MHz}$, where the HF relaxation almost diminishes. This behavior suggests that the LF and HF relaxation processes are related to the membrane polarization of the projections and the spherical body, respectively.

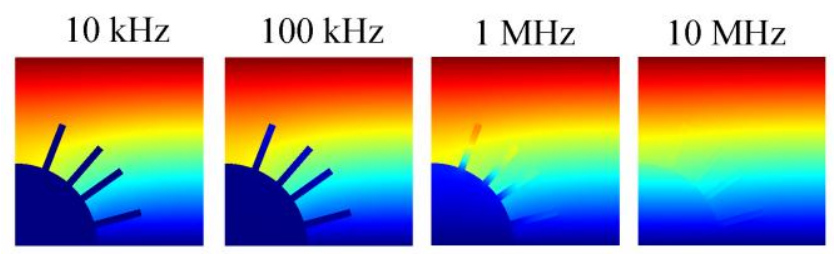

Fig. 6 Distributions of the real part of electric potential at the cross-section through points $p, q$ and $r$ shown in Fig. 1. The data are the same as those used for the dielectric spectra of model $d$ in Fig. 2. The frequency of the applied ac field is shown at the top of each panel.

\subsection{Effects of the electric properties of the projection core}

The core of a microvillus contains actin filaments that run in parallel bundle along its length. Thus, the electric properties of the projection core could be different from the cytoplasm. Here, we examine the effects of the electric properties of the projection core on the dielectric spectra of the cell suspension. Figure 7 shows the dielectric spectra obtained by changing the conductivity $\kappa_{\mathrm{pc}}$ of the projection core while the relative permittivity $\varepsilon_{\mathrm{pc}}$ of the projection core is fixed at 80 . The LF 
relaxation shifted to a lower frequency side with decreasing $\kappa_{\mathrm{pc}}$, whereas the HF relaxation was not influenced by $\kappa_{\mathrm{pc}}$. When the value of $\varepsilon_{\mathrm{pc}}$ was changed between 160 and 40, there was little influence on the dielectric spectra (data are not shown). The results also support the assignment that the LF and HF relaxation processes are attributed to the polarization of the projections and the spherical body, respectively.
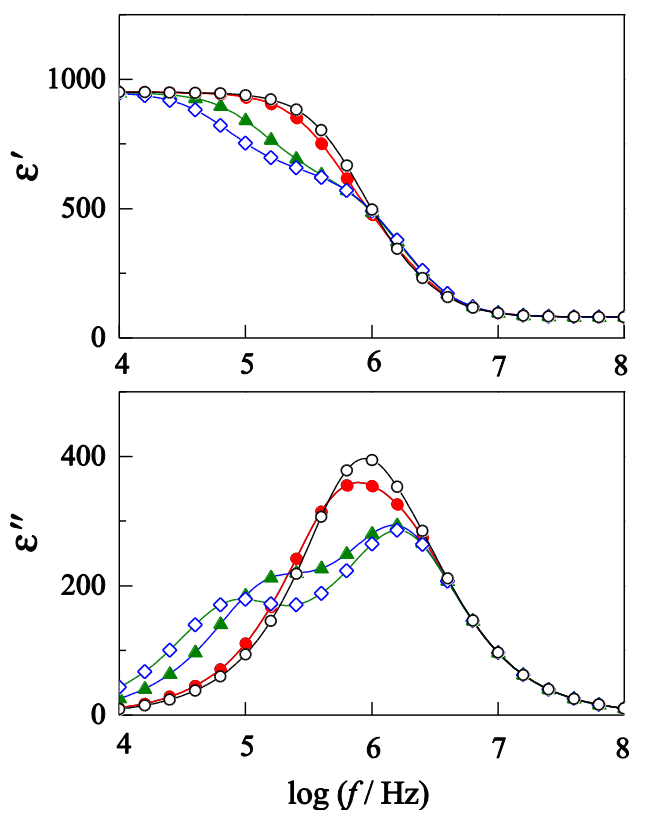

Fig. 7 The relative permittivity $\varepsilon^{\prime}$ and loss factor $\varepsilon^{\prime \prime}$ as a function of frequency $f$, which have been simulated for model $b$ when the projection core has different conductivites in $\mathrm{S} \mathrm{m}^{-1}: 1(0), 0.5(\bullet), 0.1(\boldsymbol{\Delta})$ and $0.05(\diamond)$. The other parameter values are the same as in Fig. 2

\subsection{Apparent membrane capacitance and cytoplasmic conductivity}

Dielectric spectra of spherical cell suspensions have been analyzed using the spherical shell model irrespective of cell surface morphology. In the analysis, the volume fraction $P$ of cells (or the relative volume occupied by cells in a cell suspension), the membrane capacitance $C_{\mathrm{m}}$ and the cytoplasmic conductivity $\kappa_{\mathrm{i}}$ are determined from the dielectric relaxation parameters of the dielectric spectra. The $C_{\mathrm{m}}$ of a spherical cell with a smooth surface is the specific membrane capacitance defined by $C_{\mathrm{m}}=\varepsilon_{\mathrm{m}} \varepsilon_{0} / d$, while an apparent value of $C_{\mathrm{m}}$ is obtained for a cell with a rough surface. The relationship between the value of $C_{\mathrm{m}}$ and the degree of membrane folding has not been clearly understood. In addition, the question is raised whether the surface morphology influences the estimations of $P$ and $\kappa_{\mathrm{i}}$. To answer the questions, the same analysis was applied to the simulated dielectric spectra.

When cells hold for the conditions of $d / R<<1$ and $\kappa_{\mathrm{m}}<<\kappa_{\mathrm{a}} \approx \kappa_{\mathrm{i}}$, the PS theory based on the spherical shell model predicts dielectric relaxation of a single relaxation time. The dielectric relaxation parameters $\kappa_{1}, \Delta \varepsilon, f_{\mathrm{c}}$ and $\kappa_{\mathrm{h}}$ (the high-frequency limit of conductivity) are related to $P, C_{\mathrm{m}}$ and $\kappa_{\mathrm{i}}[3,6]$ as:

$$
P=\frac{2\left(1-\kappa_{l} / \kappa_{a}\right)}{2+\kappa_{l} / \kappa_{a}}
$$




$$
\begin{gathered}
C_{m}=\Delta \varepsilon \frac{\varepsilon_{0}(2+P)^{2}}{9 P R}, \\
\kappa_{i}=\left[\frac{9 P}{2 \pi f_{c} \Delta \varepsilon \varepsilon_{0}(2+P)^{2}}-\frac{1-P}{\kappa_{a}(2+P)}\right]^{-1}, \\
\kappa_{i}=\kappa_{a} \frac{(2+P) \kappa_{h}-2(1-P) \kappa_{a}}{(1+2 P) \kappa_{a}-2(1-P) \kappa_{h}} .
\end{gathered}
$$

Analysis with Eqs. (7)-(10) was carried out for the dielectric spectra simulated for the case of $\varepsilon_{\mathrm{i}}{ }^{*}=\varepsilon_{\mathrm{pc}}{ }^{*}$ described in section 3.1. Figure 8 shows the values of $P$ calculated from Eq. (7) plotted against those of $P_{\mathrm{g}}$ calculated from the geometry of the cell model. The values of $P$ were in good agreement with those of $P_{\mathrm{g}}$, suggesting that Eq. (7) is applicable to microvillous cells at volume fractions below at least 0.13 and that the estimation of $P$ is insensitive to the cell surface morphology.

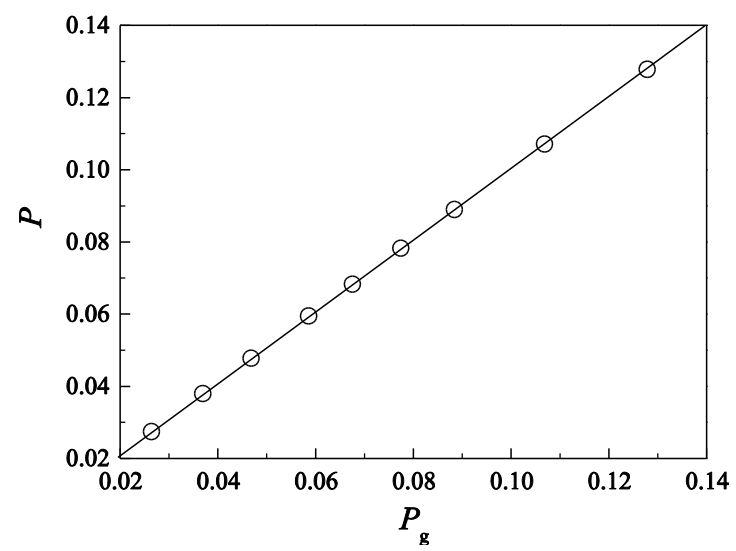

Fig. 8 The volume fraction $P$ calculated from Eq. (7) as a function of the volume fraction $P_{\mathrm{g}}$ that is the relative volume occupied by the cell model in the cubical system. A line with a slope of unity is shown.

The values of $C_{\mathrm{m}}$ were calculated from Eq. (8) with the values of $R$ and $P$. The choice of the value of $R$ is, however, arbitrary, although, in experiment, the value of $R$ is determined by optical microscopy. Here, the values of $R_{\mathrm{eq}}$ and $R_{\mathrm{s}}$ were used for $R$. Figure 9 shows double logarithmic plots of the estimated values of $C_{\mathrm{m}}$ against $R$. Linear relations were found, whose slopes were -2.27 with $R=R_{\text {eq }}$ and -2.16 with $R=R_{\mathrm{s}}$. As a fist approximation, therefore, $C_{\mathrm{m}}$ is inversely proportional to $R^{2}$ as $C_{m} \approx C_{m 0}\left(R_{0} / R\right)^{2}$, where $C_{\mathrm{m} 0}$ is the specific membrane capacitance of the spherical cell (without projection) of radius $R_{0}$. Using this relation, the cell total membrane capacitance $C_{\mathrm{t}}$ becomes $C_{t}=4 \pi R^{2} C_{m} \approx 4 \pi R_{0}^{2} C_{m 0}$. Hence, $C_{\mathrm{t}}$ is independent of $R$, which relation accounts for the experimental results that $C_{\mathrm{t}}$ remained constant irrespective of changes in $R$ with the medium osmolality $[15,16]$. In other words, if the value of $C_{\mathrm{m} 0}$ is given, the value of $C_{\mathrm{t}}$ provides the total surface area of the cell. For the value of $C_{\mathrm{m} 0}$, we may adopt a value of $6-8 \mathrm{mF} \mathrm{m} \mathrm{m}^{-2}$ obtained for erythrocyte membranes with smooth surfaces. 


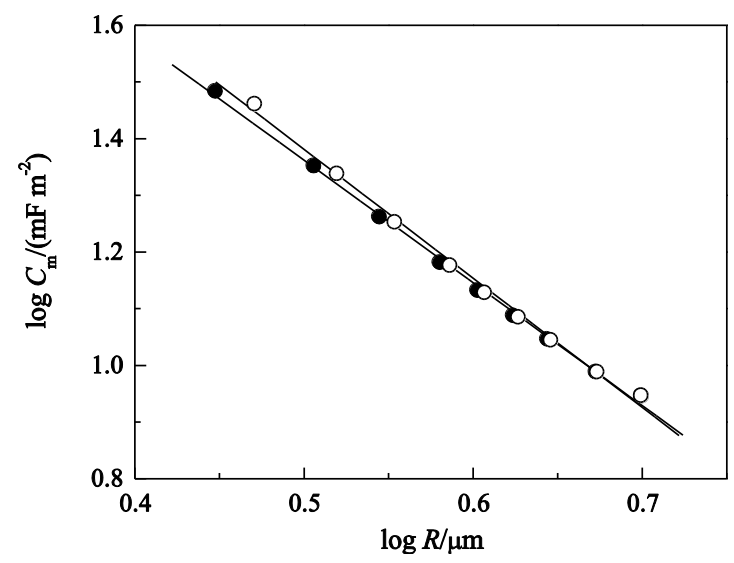

Fig. 9 The membrane capacitance $C_{\mathrm{m}}$ estimated by Eq. (8) as a function of the cell radius $R$. Data points (०) and (•) are calculated with $R=R_{\text {eq }}$ and $R_{\mathrm{s}}$, respectively. Solid lines are regression lines.

The cytoplasmic conductivity $\kappa_{\mathrm{i}}$ was estimated from the values of $f_{\mathrm{c}}$ and $\kappa_{\mathrm{h}}$ using Eqs. (9) and (10), respectively (Fig. 10). The value of $f_{\mathrm{c}}$ was approximately determined assuming a single relaxation process, although the simulated dielectric spectra contained two relaxation processes. The value of $\kappa_{\mathrm{h}}$ was obtained at $100 \mathrm{MHz}$, where the conductivity almost leveled off. The values of $\kappa_{\mathrm{i}}$ estimated from those of $f_{\mathrm{c}}$ seriously deviated from the true value $\left(0.5 \mathrm{~S} \mathrm{~m}^{-1}\right)$ at low $R_{\mathrm{eq}}$, whereas the estimation of $\kappa_{\mathrm{i}}$ from $\kappa_{\mathrm{h}}$ provided reasonable values over the whole range of $R_{\text {eq }}$. The results suggest that the estimation of $\kappa_{\mathrm{i}}$ from $f_{\mathrm{c}}$ includes serious errors for microvillous cells in contrast to that from $\kappa_{\mathrm{h}}$.

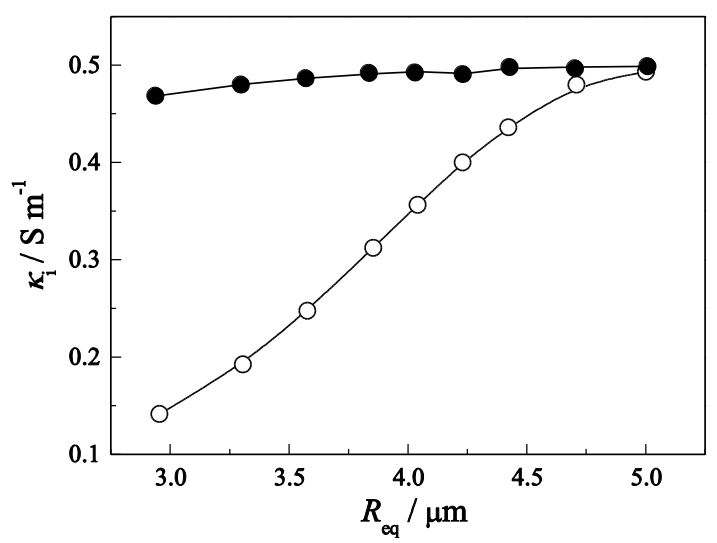

Fig. 10 Cytoplasmic conductivity $\kappa_{\mathrm{i}}$ as a function of effective cell radius $R_{\text {eq. }}$. Data points (०) and $(\bullet)$ are calculated from Eqs. (9) and (10), respectively.

\section{Conclusion}

A microvillous cell was modeled as a sphere with projections and its dielectric properties were numerically simulated by 3D-FEM. The $\varepsilon^{*}$ of the cell suspension showed dielectric dispersion consisting of LF and HF relaxation processes, which were respectively attributed to the polarization of the membranes covering the projections and the spherical body. The values of $C_{\mathrm{m}}$ were obtained from the simulated spectra of $\varepsilon^{*}$ by the conventional analysis based on the spherical shell model. When leaving the surface area of the cell model constant, the $C_{\mathrm{m}}$ was inversely proportional to the 
square of the apparent cell radius and the cell total membrane capacitance remained constant, which was consistent with previous experimental results. The estimation of $\kappa_{\mathrm{i}}$ from the value of $f_{\mathrm{c}}$ in $\varepsilon^{*}$ spectra included errors, and consistent values of $\kappa_{\mathrm{i}}$ were obtained from the value of $\kappa_{\mathrm{h}}$.

The present simulation has demonstrated that the dielectric properties of cells are seriously influenced by their surface morphology. When applying the conventional spherical shell model to microvillous cells, careful considerations are required in interpreting the estimated values of electric parameters of the plasma membrane and the cytoplasm.

Acknowledgement I thank Dr. K. Sekine for his critical reading of the manuscript and useful comments. This work was partially supported by Grant-in-Aid for Scientific Research (C) 21560447 from the Ministry of Education, Science, Sports and Culture.

\section{References}

[1] R. Pethig, D.B. Kell, The passive electrical properties of biological systems: their significance in physiology, biophysics and biotechnology, Phys. Med. Biol. 32 (1987) 933-970

[2] K.R. Foster, H.P. Schwan, Dielectric properties of tissues in: C Polk and E Postow (Eds.) Handbook of Biological Effects of Electromagnetic Fields, CRC Press, Boca Raton, 1996 pp 25-102

[3] T. Hanai, Electrical properties of emulsions, in: P Sherman (Ed.) Emulsion Science, Academic Press, London and New York, 1968, pp 353-478

[4] K. Asami, Characterization of heterogeneous systems by dielectric spectroscopy, Prog. Polym. Sci. 27 (2002) 1617-1659

[5] T.B. Jones, Electromechanics of particles, Cambridge University Press, Cambridge 1995

[6] H. Pauly, H.P. Schwan, Über die Impedanz einer Suspension von Kugelförmigen Teilchen mit einer Schale, Z. Naturforsch. 14b (1959) 125-131

[7] T. Hanai, K. Asami, N. Koizumi, Dielectric theory of concentrated suspension of shell-spheres in particular reference to the analysis of biological cell suspensions, Bull. Inst. Chem. Res., Kyoto University 57 (1979) 297-305

[8] S. Takashima, K. Asami, Y. Takahashi, Frequency domain studies of impedance characteristics of biological cells using micropipette technique. I. Erythrocyte, Biophys. J. 54 (1988) 995-1000

[9] K. Asami, Y. Takahashi, S. Takashima, Dielectric properties of mouse lymphocytes and erythrocytes, Biochim. Biophys. Acta 1010 (1989) 49-55

[10] H. Kaneko, K. Asami, T Hanai, Dielectric analysis of sheep erythrocyte ghost. Examination of applicability of dielectric mixture equations, Colloid Polym. Sci. 269 (1991) 1039-44

[11] R. Lisin, B.Z. Ginzburg, M. Schlesinger, Y. Feldman, Time domain dielectric spectroscopy study of human cells. I. Erythrocytes and ghosts, Biochim. Biophys. Acta 1280 (1996) 34-40

[12] Y. Hayashi, I. Oshige, Y. Katsumoto, S. Omori, A. Yasuda, K. Asami, Dielectric inspection of erythrocyte morphology, Phys. Med. Biol. 53 (2008) 2553-2564

[13] J. Gimsa, T. Schnelle, G. Zechel, R. Glaser, Dielectric spectroscopy of human erythrocytes: investigations under the influence of nystatin, Biophys. J. 66 (1994) 1244-1253 
[14] A. Irimajiri, K. Asami, T. Ichinowatari, Y. Kinoshita, Passive electrical properties of the membrane and cytoplasm of cultured rat basophil leukaemia cells. II. Effects of osmotic perturbation, Biochim. Biophys. Acta 896 (1987) 214-223

[15] V.L. Sukhorukov, W.M. Arnold, U. Zimmermann, Hypotonically induced changes in the plasma membrane of cultured mammalian cells, J. Membrane Biol. 132 (1993) 27-40

[16] X.B. Wang, Y. Huang, P.R.C. Gascoyne, F. F. Becker, R. Hölzel, R. Pethig, Changes in friend murine erythroleukaemia cell membranes during induced differentiation determined by electrorotation, Biochim. Biophys. Acta 1193 (1994) 330-344

[17] K. Ratanachoo, P.R.C. Gascoyne, M. Ruchirawat, Detection of cellular responses to toxicants by dielectrophoresis, Biochim. Biophys. Acta 1564 (2002) 449-458

[18] X. Wang, F.F. Becker, P.R.C. Gascoyne, Membrane dielectric changes indicate induced apoptosis in HL-60 cells more sensitively than surface phosphatidylserine expression or DNA fragmentation, Biochim. Biophys. Acta 1564 (2002) 412-420

[19] C. Huang, A. Chen, L. Wang, Electrokinetic measurements of dielectric properties of membrane for apoptotic HL-60 cells on chip-based device, Biomed. Mirodevices 9 (2007) 335-343

[20] C. Huang, A. Chen, M. Guo, Membrane dielectric responses of bufalin-induced apoptosis in HL-60 cells detected by an electrorotation chip, Biotechnol. Lett. 29 (2007) 1307-1313

[21] R. Pethig, M.S. Talary, Dielectrophoretic detection of membrane morphology changes in Jurkat T-cells undergoing etoposide-induced apoptosis, IET Nanobiotechnol. 1 (2007) 2-9

[22] F.H. Labeed, H.M. Coley, M.P. Hughes, Differences in the biophysical properties of membrane and cytoplasm of apoptotic cells revealed using dielectrophoresis, Biochim. Biophys. Acta 1760 (2006) 922-929

[23] K. Sekine, Application of boundary element method to calculation of the complex permittivity of suspensions of cells in shape of $\mathrm{D}_{\text {oh }}$ symmetry, Bioelectrochem. 52 (2000) 1-7

[24] K. Sekine, C. Hibino, M. Kimura, K. Asami, Effects of T-tubules on dielectric spectra of skeletal muscle simulated by boundary element method with two-dimensional models, Bioelectrochem. 70 (2007) 532-541

[25] K. Sekine, T. Takeda, K. Nagaomo, E. Matsushima, Boundary-element calculations for amplification of effects of low-frequency electric fields in a doublet-shaped biological cell, Bioelectrochem.77 (2010) 106-113

[26] M. Sancho, G. Martinez, C. Martin, Accurate dielectric modeling of shelled particles and cells, J. Electrostat. 57 (2003) 143-156

[27] T. R. Gowrishankar, J.C. Weaver, An approach to electrical modeling of single and multiple cells, Proc. Natl. Acad. Sci. USA 100 (2003) 3203-3208

[28] T. R. Gowrishankar, D. A. Stewart, J. C. Weaver, Model of a confined spherical cell in uniform and heterogeneous applied electric fields, Bioelectrochem. 68 (2006) 181-190

[29] A. di Biasio, C. Cametti, Effect of the shape of human erythrocytes on the evaluation of the passive electrical properties of the cell membrane, Bioelectrochem. 65 (2005) 163-169

[30] A. di Biasio, C. Cametti, Effect of shape on the dielectric properties of biological cell suspensions, Bioelectrochem. 71 (2007) 149-156

[31] A. di Biasio, L. Ambrosone, C. Cametti, Numerical simulation of dielectric spectra of aqueous suspensions of non-spheroidal differently shaped biological cells, J. Phys. D: Appl. Phys. 42 (2009) 025401 
[32] A. Ron, N. Fishelson, I. Shur, N. Croitoru, D. Benayahu, Y. Schacham-Diamand, Theoretical examination of aggregation effect on the dielectric characteristics of spherical cellular suspension, Biophys. Chem. 140 (2009) $39-50$

[33] A. Ron, N. Fishelson, I. Shur, N. Croitoru, D. Benayahu, Y. Schacham-Diamand, The effect of irregularity on the dielectric dispersion characteristics of spherical cellular suspension, Colloids and Surfaces B 74 (2009) 127-135

[34] P. Salou, A. Mejdoubi, C. Brosseau, Modeling of the dielectric relaxation in eukaryotic cells, J. Applied Phys. 105 (2009) 114702

[35] Y. Katsumoto, Y. Hayashi, I. Oshige, S. Omori, N. Kishii, A. Yasuda, K. Asami, Dielectric cytometry with three-dimensional cellular modelling, Biophys. J. 95 (2008) 3043-3047

[36] K. Asami, Dielectric dispersion in biological cells of complex geometry simulated by the three-dimensional finite difference method, J. Phys. D: Appl. Phys. 39 (2006) 492-499

[37] K. Asami, Effectiveness of "thin-layer" and "effective medium" approximations in numerical simulation of dielectric spectra of biological cell suspensions, Jpn. J. Applied Phys. 49 (2010) 127001

[38] B. Alberts, D. Bray, J. Lewis, M. Raff, K. Roberts, J.D. Watson, Molecular Biology of the Cell, Garland Publishing, New York and London, 1983 pp 582-585 